\title{
A Study of Cytohistopathological Correlation of Palpable Breast Lumps
}

\author{
${ }^{1}$ Ankita Das, ${ }^{2} \mathrm{~B}$ Niranjana Murthy
}

\begin{abstract}
Introduction: Breast diseases are showing rising trend worldwide. Benign breast disease (BBD) is the most common cause of breast problem among women with prevalence rate of $68 \%$ among all breast lesions in India. It is more frequent than malignancy. Making an early diagnosis and planning the treatment help in alleviating unnecessary anxiety about breast cancer, and those with an increased risk of malignancy like atypical hyperplasia are given prompt treatment, follow-up, and awareness regarding the risk of breast cancer. Fine needle aspiration cytology (FNAC) is a relatively simple, reliable, nontraumatic, complication-free technique for breast lump evaluation.
\end{abstract}

Materials and methods: This study was done on a total of 55 female patients attending hospital for a period of 1 year in Tumkur, Karnataka. The FNAC technique was done with 23G needle attached to $10 \mathrm{~mL}$ syringe inserted into palpable breast mass. Cellular material aspirated and smeared on glass slide, fixed in 95\% methanol, stained with hematoxylin and eosin. Air-dried slides were stained with Giemsa. Histopathological diagnosis was correlated with FNAC details in all cases.

All the collected data were transferred into a master sheet and fed into computer for statistical analysis using Pearson's Chi-square test.

Results: In our study the patients belonged to age group of 14 to 65 years with a median of 37.5 years. Irrespective of sides, maximum numbers of cases were found in upper and outer quadrants. Maximum malignant lesions were in the age group of 45 to 49 years, whereas benign lesions were maximum in 30 to 34 years age group. The commonest pathology found in our study was fibroadenoma (51\%) followed by ductal carcinoma (27\%).

In our study out of 55 patients, 2 cases where FNAC showed benign proliferative breast disease, histopathological examination (HPE) report suggested infiltrating ductal carcinoma (IDC). Another 1 case where FNAC showed fibrocystic disease with atypical ductal hyperplasia (ADH), HPE report turned it out as IDC. Sensitivity of $75 \%$ specificity $100 \%$, positive predictive value (PPV) $100 \%$, and negative predictive value of $93 \%$ were obtained.

Conclusion: Fine needle aspiration cytology is a very important preliminary diagnostic tool in palpable breast lumps and results show high degree of correlation with final HPE report.

\footnotetext{
${ }^{1}$ Junior Resident, ${ }^{2}$ Professor and Head

1,2Department of Pathology, Sri Siddhartha Medical College and Research Centre, Tumkur, Karnataka, India
}

Corresponding Author: Ankita Das, Junior Resident Department of Pathology, Sri Siddhartha Medical College and Research Centre, Tumkur, Karnataka, India, e-mail: drankitadas 1990@gmail.com
Keywords: Breast disease, Fine needle aspiration cytology, Histopathology.

How to cite this article: Das A, Murthy BN. A Study of Cytohistopathological Correlation of Palpable Breast Lumps. J Med Sci 2018;4(2):52-56.

\section{Source of support: Nil}

\section{Conflict of interest: None}

\section{INTRODUCTION}

Breast is an organ that undergoes cyclical changes under the influence of various hormones throughout a woman's reproductive life. Breast diseases are showing rising trend worldwide. Wide spectrum of disorders ranging from self-limiting inflammatory lesion, benign breast lesion to life-threatening invasive carcinoma arises from breast.

Benign breast diseases constitute a heterogeneous group of disorders, including developmental abnormality, epithelial and stromal proliferations, inflammatory lesions, and neoplasm. It is the most common cause of breast problem among women with prevalence rate of $68 \%$ among all breast lesions in India. It starts to rise in second decade of life, peaks in third decade, and decline thereafter, whereas incidence of breast carcinoma rises as age advances. It is more frequent than malignancy. Benign breast lesions deserve attention because of their high prevalence, their impact on women's life, and due to cancerous potential of some histological types. ${ }^{1}$

Benign breast disease is more frequent than malignant ones. Up to $30 \%$ women who suffer from BBDs will require treatment at some point in their lives. ${ }^{2}$ Benign neoplasms include fibroadenoma, benign phyllodes, tubular adenoma, duct papilloma, fibrocystic disease, sclerosing adenosis, granulomatous mastitis, tuberculous mastitis, and fat necrosis.

Breast cancer is the most common female cancer worldwide, representing nearly a quarter $(25 \%)$ of all cancers. Cancer breasts constitute 14.3 to $30 \%$ of all cancers in women (as per the data collected from hospitalbased cancer registry). The data from the report of the project on "Development of an Atlas of Cancer in India" show that, in Hospital-based Cancer Registry, cancer of the breast is the leading site of cancer in Mumbai and in Thiruvananthapuram. The second leading states include Bengaluru and Chennai. ${ }^{3}$ As per the statistics reported 
in GLOBOCAN, World Health Organization 2012 (International Agency for Research on Cancer) in India, 144,937 women were newly detected with breast cancer and 70,218 women died of breast cancer. ${ }^{4}$ So, roughly for every two women newly diagnosed with breast cancer, one lady is dying of it. The reported incidence rates for breast cancer from the National Cancer Registry Programme (NCRP) data indicate that percentage of breast cancer relative to total cases over time in Bengaluru and Chennai has increased. ${ }^{4}$ Also, projections of cancer cases in India (2010-2020) of NCRP indicate that there will be more than 100,000 estimated number of breast cancer cases annually in India based on the data from NCRP in Bengaluru. ${ }^{5}$ Cancer breast has emerged as leading site of cancer in most urban populations of India. It is rapidly replacing cancer of cervix as most important leading site of cancer among women. ${ }^{3}$ Several etiological factors, such as age, genetics, family history, diet, alcohol, obesity, lifestyle, physical inactivity, endocrine factors, are implicated in pathogenesis of disease. Current management of carcinoma of the breast is multimodality treatment, which includes surgery, radiotherapy, chemotherapy, and hormone therapy.

Malignant tumors include IDC not otherwise specified (NOS), metaplastic carcinoma, mucinous carcinoma, invasive lobular carcinoma (ILC), invasive papillary carcinoma, intracystic papillary carcinoma. The IDC-NOS is the most common malignant neoplasm.

A triple assessment, which is done by a clinical examination imaging like ultrasonography or mammography and a pathological examination-FNAC, during the initial consultation allows a majority of the patients with discrete BBDs to be given immediate reassurance. Making an early diagnosis and planning the treatment within 72 hours of the first consultation help in alleviating unnecessary anxiety about breast cancer, and those BBDs patients with an increased risk of malignancy like atypical hyperplasia are given a prompt treatment, a proper follow-up, and awareness regarding the risk of breast cancer. $^{2}$ Fine needle aspiration cytology is a relatively simple, reliable, nontraumatic, complication-free technique for breast lump evaluation. It has reduced number of open biopsies because of high diagnostic sensitivity and specificity. Most countries have adopted triple assessment approach to breast diagnosis with FNAC included as one of pathological investigations.

\section{MATERIALS AND METHODS}

This retrospective study was done on a total of 55 female patients attending hospital for a period of 1 year in Tumkur, Karnataka. After taking consent, detailed history of patient, including past history and family history, thorough physical examination of the patient, was done to find out regarding position, size, shape, mobility, and consistency of palpable breast mass.

Fine needle aspiration cytology was done with 23G needle attached to $10 \mathrm{~mL}$ syringe inserted into palpable mass. Cellular material aspirated and smeared on glass slide, fixed in $95 \%$ methanol, stained with hematoxylin and eosin. Air-dried slides were stained with Giemsa. Histopathological diagnosis was also correlated with FNAC details in all cases.

Biopsy specimens were received in $10 \%$ formalin. In cases where mastectomy was done whole breast mass was received and grossing was done as per standard procedures. Specimens were then systematically examined. Representative tissue section processed routinely by paraffin section for light microscopy.

Benign tumors included fibroadenoma, phyllodes tumor, fibrocystic disease, papillomas, tubular adenoma, lactating adenoma, and epithelial hyperplasia.

Malignancy included ADH, atypical lobular hyperplasia, carcinoma in situ (ductal carcinoma in situ, lobular carcinoma in situ), and infiltrating carcinomas (IDC-NOS, ILC, tubular carcinoma, medullary carcinoma, mucinous carcinoma, invasive papillary carcinoma, invasive micropapillary carcinoma, apocrine carcinoma, secretory carcinoma, etc.).

\section{Statistical Analysis}

All collected data were transferred into a master sheet and fed into computer for statistical analysis. Data were analyzed using Statistical Package for the Social Sciences program. Pearson's Chi-squared test was used for statistical analysis.

\section{RESULTS}

In our study, a total of 55 cases were studied for cytohistological correlation of breast lumps. In our study (Graph 1), the patients belonged to age group of 14 to 65 years with a median of 37.5 years. Irrespective of sides, maximum numbers of cases were found in upper and outer quadrants. Maximum malignant lesions were in the age group of 45 to 49 years, whereas benign lesions were maximum in 30 to 34 years age group. The commonest pathology found in our study was fibroadenoma (51\%) (Fig. 1) followed by ductal carcinoma (27\%).

In our study out of 55 patients, 2 cases where FNAC showed benign proliferative breast disease, HPE report suggested IDC. Another 1 case where FNAC showed fibrocystic disease with ADH, in HPE report it turned out to be IDC. Thus, we got 9 true positive, 43 true negative, 0 false positive, and 3 false negative. The statistical tests used in interpretation of results obtained in our study 


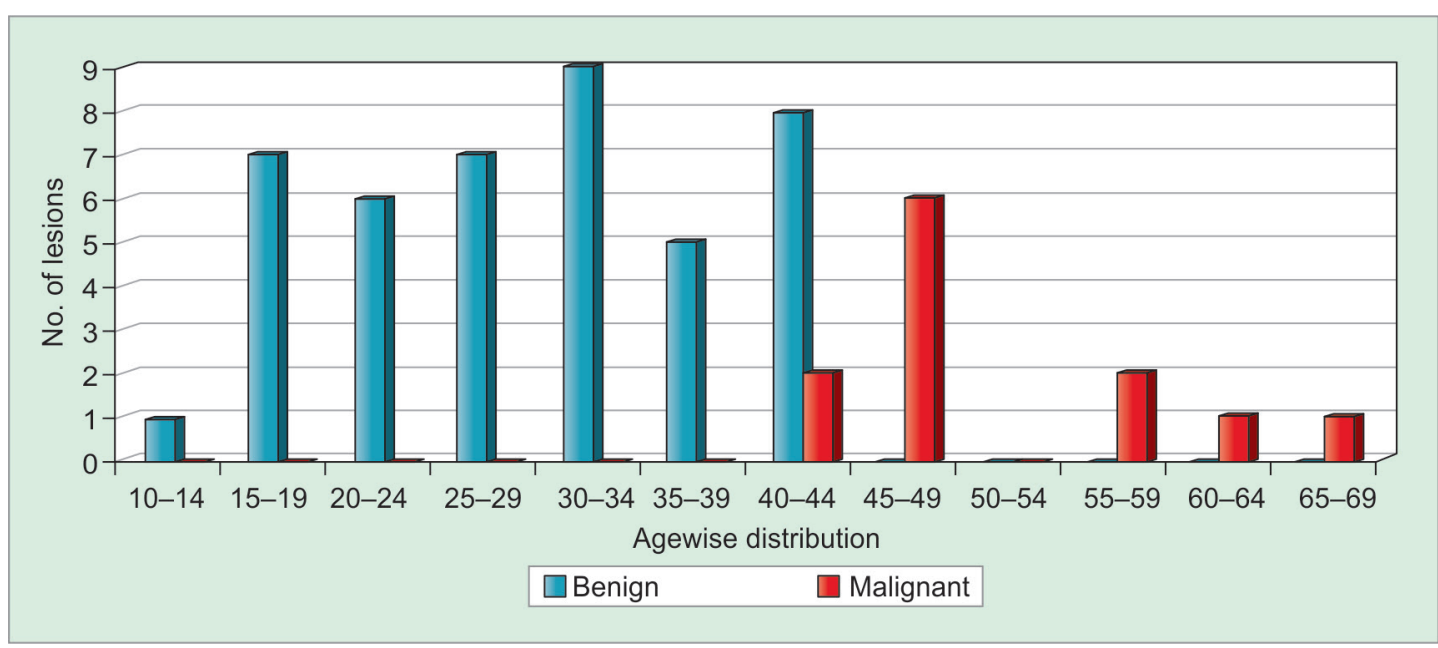

Graph 1: Age-wise distribution of lesions
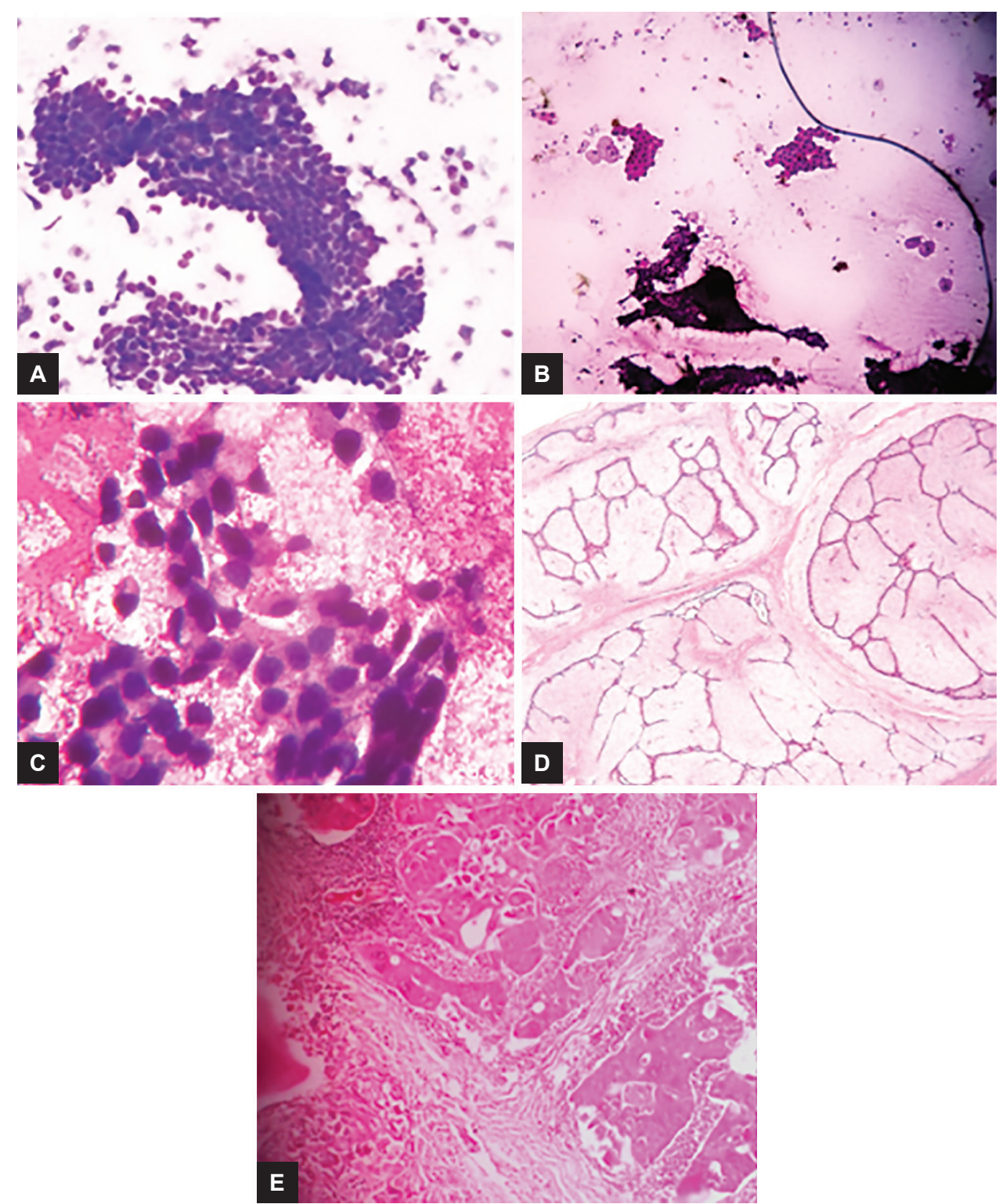

Figs 1A to E: (A) FNAC: Fibroadenoma; (B) fibrocystic disease with apocrine cells; (C) carcinoma; (D) HPE: Fibroadenoma; (E) IDC-NOS 
are: sensitivity: 75\%, specificity: 100\%, PPV: 100\%, and negative predictive value: $93 \%$.

\section{DISCUSSION}

In our study, benign lesions were commonest in the age group of 30 to 34 years and malignant lesions were commonest in 45 to 49 years. Many studies (Table 1) concluded that the incidence of benign breast lesions begins to rise during the second decade of life and peaks in the fourth decade. This is because changes in breast are most dynamic and profound during reproductive years of female. Before pregnancy lobules are small and invested by loose cellular intralobular stroma and during pregnancy branching of terminal ducts produce numerous larger lobules. Both epithelial and stromal elements of lobule are under hormonal control. Any interference with these close interactions results in conditions grouped under BBD. Also, in repeated development and involutional changes of menstruation, pregnancies are responsible for minor aberrations. In 2015, Pandey et al conducted a study of 781 patients with breast pathologies at Department of Surgery, Mahatma Gandhi Institute of Medical Sciences, Sevagram, Wardha, Maharashtra. They observed that out of 781 patients, 722 had BBD accounting for $92.4 \%$. In another study, BBDs were more common in 21 to 30 years age followed by 31 to 40 and 11 to 20 years. ${ }^{6}$

Kapoor et $\mathrm{al}^{6}$ observed malignant lesions were common in fourth decade followed by fifth, sixth, seventh decade. Mudholkar et $\mathrm{al}^{7}$ observed in his study that maximum cases were found in fifth decade. Similar findings were reported by Bhalla et $\mathrm{al}^{8}$ and Kalyani and Banu. ${ }^{9}$ Incidence of breast cancer increases as age advances peaking at 70 to 80 years. Late menopause results in prolonged exposure to estrogens that increase risk. With increasing age breast tissue gets

Table 1: Supporting studies

\begin{tabular}{lll}
\hline Studies & Benign (years) & Malignant (years) \\
\hline${\text { Bhalla et } \mathrm{al}^{8}}^{8}$ & $17-55$ & $23-75$ \\
${\text { Mudholkar et } \mathrm{al}^{7}}_{\text {Kalyani and Meharaj Banu }^{9}}$ & $11-60$ & $27-80$ \\
Kapoor et al $^{6}$ & $11-40$ & $31-70$ \\
\hline
\end{tabular}

replaced by adipose tissue and fibrous connective tissue increases in density. Women with very dense breasts have increased risk of carcinoma. Extensive mammographic density and delayed breast involution, both result in cumulative exposure of breast tissue to hormones and growth factors that stimulate cell division and accumulation of genetic damage in breast cells that are major determinants of breast cancer incidence. The risk for breast cancer also rises with increasing severity of BBD, including hyperplasia with moderate to marked atypia in premenopausal women. Thus, the present study is in concordance with the studies available in the literature.

The commonest pathology found in our study (Table 2) was fibroadenoma (51\%) followed by ductal carcinoma $(27 \%)$. Our finding was in agreement with most of available literature on benign breast lumps where frequency of fibroadenomas ranged from 46.6 to $55.6 \%$. Peak incidence of fibroadenoma ranged from second to third decade of life. Since they arise from lobules they are seen predominantly in 15 to 25 years age group. Fibroadenomas also represent "aberrations of normal development and involution." Clinical observations in women receiving estrogens suggest hormonal events play a role in etiology. A direct association has been noted between oral contraceptive usage before 20 years age and risk of fibroadenoma. In malignancy, commonest neoplasm was IDC-NOS (85\%). The findings in our study correlated with others like Mudholkar et al, ${ }^{7}$ Bhalla et al, ${ }^{8}$ Kapoor et al, ${ }^{6}$ Rasheed, ${ }^{10}$ and Kalyani and $\mathrm{Banu}^{9}$ that have reported a low incidence of 19.44 and $20.15 \%$ of IDC-NOS respectively. Kalyani and Banu (Table 1) studied 129 cases over a period of 1 year of which maximum were benign lesions $77.5 \%$ and only $22.5 \%$ malignant lesions, which could be the reason for low incidence.

In the present study, 55 cases had cytohistological investigation done. Out of 55 cases, 52 cases showed cytohistological correlation, rest 3 cases showed discrepancy (Table 3). In our study, 2 cases of benign proliferative breast disease on FNAC turned out to be IDC-NOS on biopsy and 1 case of fibrocystic disease with $\mathrm{ADH}$ on

Table 2: Cytohistological correlation of cases

\begin{tabular}{lllll}
\hline Diagnosis & $\begin{array}{l}\text { No. of cases } \\
\text { diagnosed in FNAC }\end{array}$ & $\begin{array}{l}\text { No. of cases consistent } \\
\text { with HPE }\end{array}$ & $\begin{array}{l}\text { No. of cases inconsistent } \\
\text { with HPE }\end{array}$ & $\begin{array}{l}\text { Improved } \\
\text { diagnosis }\end{array}$ \\
\hline Inflammatory & 2 & 2 & - & - \\
Fibroadenoma & 30 & 30 & - & - \\
Fibrocystic & 7 & 7 & - & - \\
BPBD & 2 & - & 2 & IDC \\
Benign phyllodes & 4 & 4 & - & - \\
Fibrocystic disease with ADH & 1 & - & 1 & IDC \\
Ductal carcinoma & 9 & 9 & - & - \\
\hline
\end{tabular}


Table 3: Discrepant cases

\begin{tabular}{llll}
\hline HPE & Malignant & Nonmalignant & Total \\
\hline Malignant & 9 & - & 9 \\
Nonmalignant & 3 & 43 & 46 \\
\hline Total & 12 & 43 & 55 \\
\hline
\end{tabular}

Table 4: Studies supporting our findings of specificity, sensitivity, PPV

\begin{tabular}{|c|c|c|c|}
\hline Studies & Sensitivity (\%) & Specificity (\%) & $P P V(\%)$ \\
\hline Ahmed et al ${ }^{12}$ & 92.6 & 95.2 & 95.5 \\
\hline Yalavarthi et $\mathrm{al}^{13}$ & 100 & 88.5 & 84 \\
\hline Kanchana et $\mathrm{al}^{14}$ & 87.5 & 100 & 100 \\
\hline Usman et al ${ }^{15}$ & 85.96 & 88.81 & 75.38 \\
\hline
\end{tabular}

FNAC turned out to be IDC-NOS on biopsy. In our study, we missed the diagnosis due to small size of the tumor that was obscured by surrounding fibrous stroma and in other case tumor was situated adjacent to a benign cystic lesion that was missed by the needle. In third case, it was a small tumor situated deep with cystic change next to tumor area. Sampling from representative area was missed. Infiltrating ductal carcinoma on cytology can appear fibrocystic due to FNAC done from nonrepresentative area. So, image guidance is important to increase diagnostic accuracy in smaller deeper lesions. The findings of Dey and Luthra ${ }^{11}$ indicate presence of associated fibrocystic disease can be misleading because it can mask malignancy. Hypocellularity and mild nuclear atypia are most common reasons for misdiagnosis in malignancy. In our study, sensitivity $75 \%$, specificity $100 \%$, PPV $100 \%$, negative predictive value $93 \%$ were obtained, which correlated with other studies. Results compared with other studies substantiate findings of our series. The results have been summarized in Table 4

\section{CONCLUSION}

Fine needle aspiration cytology is a sensitive and specific modality that assist in diagnosis and management of breast lesions. In conclusion, FNAC is a very important preliminary diagnostic tool in palpable breast lumps and results show high degree of correlation with final HPE report.
Thus, FNAC is a simple safe diagnostic procedure in workup of breast lesions providing enough clinical information of underlying benign or malignant pathological condition.

\section{REFERENCES}

1. Hatim K, Laxmikant N, Mulla T. Patterns and prevalence of benign breast disease in Western India. Int J Res Med Sci 2017;5(2):684-688.

2. Sangma M, Panda K, Dasiah S. A clinico-pathological study on benign breast diseases. J Clin Diagn Res 2013 Mar;7(3):503-506.

3. Nandakumar A, Ramnath T, Chaturvedi M. The magnitude of cancer breast in India: a summary. Indian J Surg Oncol 2010 Jan;1(1):8-9.

4. Saranath D, Khanna A. Current status of cancer burden: global and Indian scenario. Biomed Res J 2014;1(1):1-5.

5. Babu GR, Lakshmi SB, Thiyagarajan AT. Epidemiological correlates of breast cancer in south India. Asian Pac J Cancer Prev 2013;14(9):5077-5083.

6. Kapoor S, Kumar A, Singh A, Singh H, Singla R. Varied pattern of breast diseases-a study of 443 Cases. IOSR J Dent Med Sci 2016;15(3):36-49.

7. Mudholkar GV, Mashal NS, Kawade BS. Histopathological study of neoplastic lesions of breast. Ind Med Gazette 2012;45:118-122.

8. Bhalla A, Manjari M, Kahlon K, Kumar P, Kalra N. Cytokeratin $5 / 6$ expression in benign and malignant breast lesions. Indian J Pathol Microbiol 2010 Oct-Dec;53(4):676-680.

9. Kalyani S, Meharaj Banu OA. Study of breast lesions in the tertiary health care centre. Int J Recent Trends Sci Tech 2016;18(3):415-419.

10. Rasheed A. A three year study of breast lesions in women aged 15-70 years in a tertiary care hospital. Sch J App Med Sci 2014;2(1):166-168.

11. Dey P, Luthra UK. False negative cytologic diagnosis of breast carcinoma. Acta Cytol 1999 Sep-Oct;43(5):801-805.

12. Ahmed H, Ali SA, Almobarak OA. Utility of fine-needle aspiration as a diagnostic technique in breast lumps. Diag Cytopathol 2009 Dec;37(12):881-884.

13. Yalavarthi S, Tanikella R, Prabhala S, Tallam US. Histopathological and cytological correlation of tumors of breast. Med J DY Patil Univ 2014;7:326-331.

14. Kanchana N, Ramalaxmi B, Reddy K, Raiza D. Cytohistological correlative study of breast lesions. J Evol Med Dent Sci 2015;4(26):4418-4428.

15. Usman K, Nisar B, Sajid M. Diagnostic accuracy of fine needle aspiration cytology in a breast lump using histopathology as gold standard. PJMHS 2015;9(1):343-347. 